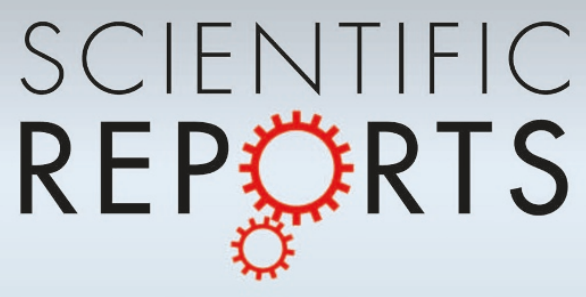

OPEN

SUBJECT AREAS:

ELECTRONIC DEVICES

ELECTRONIC PROPERTIES AND

MATERIALS

SPINTRONICS

APPLIED PHYSICS

Received

15 January 2013

Accepted

28 May 2013

Published

11 June 2013

Correspondence and requests for materials should be addressed to J.H.D. (jan-hugo.dil@ psi.ch)

\section{Tuning of the Rashba effect in Pb quantum well states via a variable Schottky barrier}

\author{
Bartosz Slomski ${ }^{1,2}$, Gabriel Landolt ${ }^{1,2}$, Gustav Bihlmayer ${ }^{3}$, Jürg Osterwalder ${ }^{1}$ \& J. Hugo Dil ${ }^{1,2}$
}

\begin{abstract}
'Physik-Institut, Universität Zürich, Winterthurerstrasse 190, CH-8057 Zürich, Switzerland, ${ }^{2}$ Swiss Light Source, Paul Scherrer Institut, CH-5232 Villigen, Switzerland, ${ }^{3}$ Peter Grünberg Institut and Institute for Advanced Simulation, Forschungszentrum Jülich and JARA, 52425 Jülich, Germany.
\end{abstract}

Spin-orbit interaction (SOI) in low-dimensional systems results in the fascinating property of spin-momentum locking. In a Rashba system the inversion symmetry normal to the plane of a two-dimensional (2D) electron gas is broken, generating a Fermi surface spin texture reminiscent of spin vortices of different radii which can be exploited in spin-based devices. Crucial for any application is the possibility to tune the momentum splitting through an external parameter. Here we show that in $\mathrm{Pb}$ quantum well states (QWS) the Rashba splitting depends on the Si substrate doping. Our results imply a doping dependence of the Schottky barrier which shifts the Si valence band relative to the QWS. A similar shift can be achieved by an external gate voltage or ultra-short laser pulses, opening up the possibility of terahertz spintronics.

T he Rashba effect ${ }^{1}$ has been studied quantitatively on a variety of high- $Z$ metal containing surfaces because of the accessibility of the surface states for spin- and angle-resolved photoemission (SARPES) experiments ${ }^{2}$. Although several surfaces show very large spin splittings, most of them have metallic substrates and are thus not suitable for the design of a spin-based devices ${ }^{3,4}$ due to the short-circuiting via the bulk states. Recently it was found that the surface of a topological insulator can also host Rashba-type spin split states that coexist with the non-trivial states ${ }^{5,6}$. However, the origin of these states is likely due to adsorption processes and an increased interlayer spacing, and they are not susceptible to a small electric field ${ }^{7}$. One of the main reasons why it is difficult to control the Rashba effect in surface or interface states by an external electric field is that this field should act directly on the states themselves and thus fields in the order of $10^{13} \mathrm{Vm}^{-1}$ are required for any substantial change ${ }^{8}$. For semiconductor heterostructures, on the other hand, the applied gate voltage indirectly affects the states via the symmetry of the confinement, and much lower voltages are required for a measurable change $e^{9-11}$.

Here we propose that quantum well states (QWS) in an ultra-thin metal film grown on a semiconductor may offer an alternative way to realize a system where the Rashba effect can be manipulated by a small gate voltage. Measured momentum splittings are larger than in semiconductor heterostructures, indicating that much shorter channel lengths are required to achieve a similar spin-manipulation or to make a conversion between charge and spin currents. We focus on $\mathrm{Pb}$ films on $\mathrm{Si}(111)$ samples with different doping levels as a model system, because (i) $\mathrm{Pb}$ is a high-Z element $(\mathrm{Z}=82)$ with large atomic SOI, (ii) smooth crystalline films can be grown on $\mathrm{Si}(111)$ with chemically sharp and well-defined interfaces ${ }^{12,13}$, and (iii) the electric field in the depletion layer due to the ionized donors and acceptors can be varied by a gate voltage.

Recently it was shown that QWS in thin Pb films on n-type Si(111) show a Rashba-type spin splitting which builds up throughout the whole metal layer along the growth direction as a result of competing effects between the metal-substrate and metal-vacuum interfaces ${ }^{14}$. This is indicated by the absence of a strong dependence of the strength of the Rashba effect on the film thickness, and by the reversed spin helicity as compared to the surface state on $\mathrm{Au}(111)^{15}$. This is in contrast to other ultra-thin film systems where the spin splitting of QWS is induced by a high- $Z$ metal substrate ${ }^{16,17}$, or by hybridization gaps ${ }^{18}$. Later the sensitivity of the Rashba parameter to the film-substrate interface was determined in $\mathrm{Pb} \mathrm{QWS}$ : replacing the $\mathrm{Pb}$ wetting layer by a Bi layer reduces the Rashba parameter by $60 \%$, whereas QWS in $\mathrm{Pb}$ films grown on a $\mathrm{Ag}$ reconstructed $\mathrm{Si}$ substrate show no measurable Rashba-type spin splitting ${ }^{19}$. As will be explained below, these effects can be understood in terms of the local asymmetry of the QWS wave function with respect to $\mathrm{Pb}$ nuclei, which in turn is related to the particular confinement conditions. Here we will show that a similar effect can be produced by changing the donor concentration of the substrate which subsequently alters the Schottky barrier (SB) of the metal-semiconductor junction. 


\section{Results}

Figure 1(a) displays ARPES data from QWS formed in an $\mathrm{N}=8$ monolayers (ML) thick $\mathrm{Pb}$ film deposited on lightly (upper panel) and heavily (lower panel) n-doped $\mathrm{Si}$ substrates [henceforth $\mathrm{Pb} / \mathrm{h}$ $\mathrm{Si}(111)$ and $\mathrm{Pb} / \mathrm{l}-\mathrm{Si}(111)]$. The band dispersions are very similar to those obtained for $\mathrm{Pb}$ films grown on a moderately $\mathrm{n}$-doped $\mathrm{Si}(111)$ substrate [hereafter $\mathrm{Pb} / \mathrm{m}-\mathrm{Si}(111)]^{20-22}$. Both band dispersions feature an anomalously high effective mass $\left(>10 \mathrm{~m}_{e}\right)$, which is due to an increased in-plane lattice constant ${ }^{23}$. The films on both samples were measured with the COPHEE spectrometer ${ }^{24}$ equipped with two Mott detectors, see Fig. 1(b), and were prepared by the same procedure. A change of the spin splitting is therefore directly related to the influence of the donor concentration in the Si substrate, because the metal-vacuum boundaries are the same. Figure 2 shows SARPES data from a Pb QWS grown on the lightly n-doped Si(111) measured for two energy distribution curves (EDCs) at (a) $\mathrm{k}_{\|}=-0.11 \AA^{-1}$ and (b) $\mathrm{k}_{\|}=-0.24 \AA^{-1}$, see also dashed arrows in the upper panel of Fig. 1(a) which indicate the positions where the spin-resolved spectra were recorded. For the measurements at both momenta displayed in Figs. 2(c, d) the spin polarization data show typical up-down excursions in the tangential spin polarization components as expected for a Rashba system.

A quantitative analysis of the spin-resolved data using a two-step fitting routine ${ }^{25}$ reveals a larger spin splitting between the spin-up $(\uparrow)$ and spin-down $(\downarrow)$ bands $\left(\Delta E:=E_{b}^{\downarrow}\left(k_{\|}\right)-E_{b}^{\uparrow}\left(k_{\|}\right)\right)$for the state with higher momentum. The corresponding fits are shown in Figs. 2(a, c) for $\mathrm{k}_{\|}=-0.11 \AA^{-1}$ and in (b, d) for $\mathrm{k}_{\|}=-0.24 \AA^{-1}$. We obtain a spin splitting of $\Delta E=-(22.2 \pm 1.4) \mathrm{meV}$ and $-(33.8 \pm 1.4) \mathrm{meV}$, respectively, with fully polarized bands. The increased spin splitting with increasing momentum is in full agreement with the Rashba model $^{1}$ where $\Delta E \propto \mathrm{k}_{\|}$, and also seen in Figs. 2(g) and (h), which display raw data as spin-resolved EDCs projected onto the quantization axis at both momenta, calculated as $I_{\tan }^{\uparrow, \downarrow}=\frac{1}{2} I_{\text {tot }}\left(1 \pm P_{\tan }\right)$ with $P_{\tan }=\operatorname{sign}\left(P_{y}\right) \sqrt{P_{x}^{2}+P_{y}^{2}}$. Figures $2(\mathrm{e})$ and (f) indicate the precise parallel momentum positions where the two EDCs were measured. For $k_{\|}=-0.11 \AA^{-1}$ the position was $\left(k_{x}, k_{y}\right)=(-0.086,0.072) \AA^{-1}$ (Fig. 2(e)), for $k_{\|}=-0.24 \AA^{-1}$ it was $\left(k_{x}, k_{y}\right)=(-0.227,0.072) \AA^{-1}$ (Fig. 2(f)). The strict Rashba-type spin-momentum locking requires the spins to be quantized in the direction perpendicular to the momenta, and the observed polarization along the $\mathrm{x}$-direction is thus a consequence of the non-zero $k_{y}$ component.
Figures 3(a-b) show SARPES data at $k_{||}=-0.24 \AA^{-1}$ for a Pb film of the same thickness prepared on the heavily n-doped $\mathrm{Si}(111)$. The best self-consistent fit to the intensity and spin polarization data is here achieved with $\Delta E=-(48.0 \pm 1.8) \mathrm{meV}$. A direct comparison of both systems at $k_{\|}=-0.24 \AA^{-1}$ reveals a larger energy splitting on the heavily $\mathrm{n}$-doped substrate by almost $14 \mathrm{meV}$, which is well beyond the combined accuracy of $2 \mathrm{meV}$ of the measurement and the fitting routine ${ }^{25}$. To illustrate that the larger $\Delta E$ of the QWS in $\mathrm{Pb} /$ $\mathrm{h}-\mathrm{Si}(111)$ is not an artifact from our analysis procedure, we have performed a fit by keeping $\Delta E$ equal to the value found in $\mathrm{Pb} / \mathrm{l}$ $\mathrm{Si}(111)$ (dashed lines in Fig. 3(a, b)). Although the total intensity fit is reasonably good, the fit to the polarization data obviously fails.

From these and further data sets measured at different $k_{\|}$we can deduce the Rashba constants for both doping levels by plotting $\Delta E$, obtained from the fitting, vs. $k_{\|}$(Fig. 3(c)). Values for $\alpha_{\mathrm{RB}}$ obtained by using $\alpha_{\mathrm{RB}}=1 / 2 \cdot(\mathrm{d} \Delta E) /\left(\mathrm{d} k_{\|}\right)$are $\alpha_{\mathrm{RB}, \mathrm{h}}=(0.11 \pm 0.007) \mathrm{eV} \AA$ for $\mathrm{Pb} /$ $\mathrm{h}-\mathrm{Si}(111), \alpha_{\mathrm{RB}, \mathrm{m}}=(0.076 \pm 0.005) \mathrm{eV} \AA$ for $\mathrm{Pb} / \mathrm{m}-\mathrm{Si}(111)^{19}$, and $\alpha_{\mathrm{RB}, \mathrm{l}}$ $=(0.070 \pm 0.011) \mathrm{eVA}$ for $\mathrm{Pb} / \mathrm{l}-\mathrm{Si}(111)$. For $\mathrm{Pb}$ on a highly $\mathrm{p}$-doped $\mathrm{Si}(111)$ sample we obtain $\alpha_{\mathrm{RB}, \mathrm{l}}=(0.061 \pm 0.006) \mathrm{eV \AA}[\mathrm{Pb} / \mathrm{p}-\mathrm{Si}(111)$, $N_{A}=1.18 \cdot 10^{16} \mathrm{~cm}^{-3}$, see SOM].

\section{Discussion}

We now discuss the mechanism leading to the increase of the Rashba constant in $\mathrm{Pb}$ QWS on the n-doped $\mathrm{Si}(111)$ with increasing donor concentration as shown in the right panel of Fig. 3(d). As demonstrated in Ref. 19 by the comparison of the Rashba effect in Pb QWS on $\mathrm{a} \mathrm{Pb}$ and $\mathrm{Bi}$ terminated $\mathrm{Si}$ surface, a changed Rashba constant results from a modified charge density distribution in the $\mathrm{Pb}$ film which alters the local asymmetric features of the QWS wave function around the $\mathrm{Pb}$ cores. According to the phase accumulation model the charge density distribution of a QWS is controlled by the phase shifts at the metal-substrate and metal-vacuum interfaces ${ }^{26}$. Because the phase shift at the vacuum side is the same for all three investigated systems, we focus in the following on the metal-substrate interface with its phase shift given by $\Phi_{S} \propto \sqrt{E-E_{0}} \Theta\left(E-E_{0}\right)^{13}$ where $E$ is the energy of a QWS and $E_{0}$ is the valence band edge of Si at $\bar{\Gamma}$ and $\Theta$ is the Heaviside function. States with energies inside the gap of a substrate are truly confined, while states outside the gap can couple to substrate states leading to quantum well resonances with a considerable fraction of charge spilling into the substrate. In between these two cases the confinement, i.e. the degree of localization, changes as a function of $E_{0}$. The goal of the following discussion is to show that the

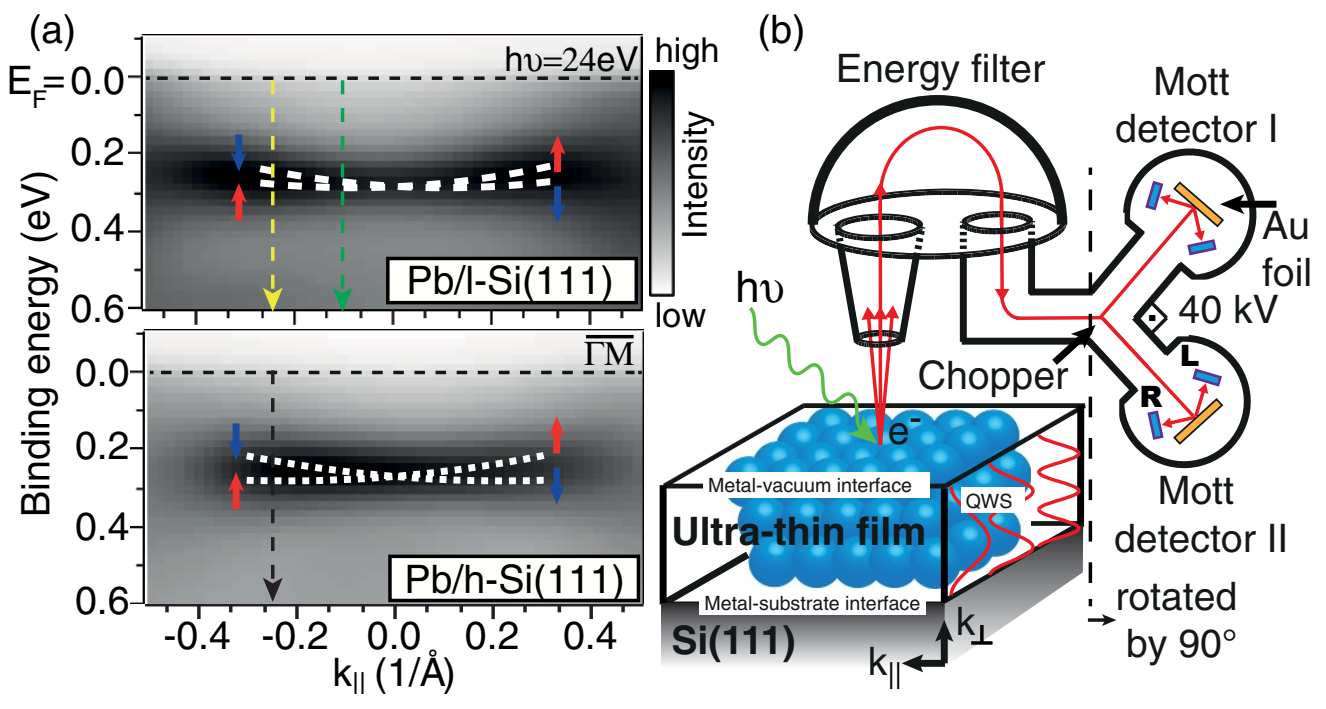

Figure $1 \mid$ (a) Band dispersion of a QWS in $8 \mathrm{ML}$ thick $\mathrm{Pb}$ films on (upper panel) lightly n-doped and (lower panel) heavily n-doped $\mathrm{Si}(111)$ substrates as measured by ARPES. (b) Schematic drawing of our SARPES experiment. 

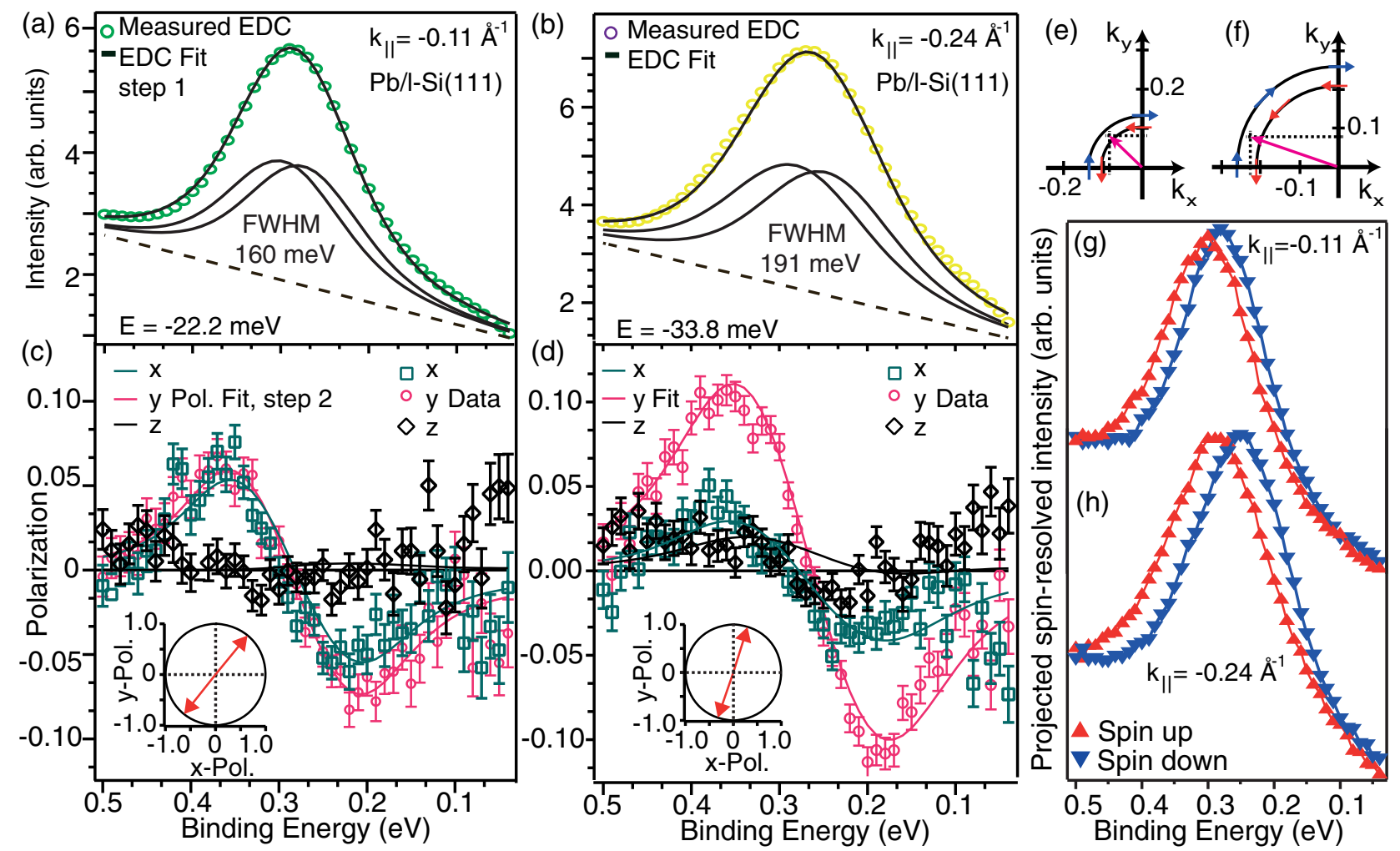

Figure $2 \mid$ Measured spin-integrated EDCs for Pb/l-Si(111) (a) at $\mathrm{k}_{\|}=-0.11 \AA^{-1}$ and at (b) $\mathrm{k}_{\|}=-0.24 \AA^{-1}$ with Voigt profiles obtained from the selfconsistent two-step fitting routine. (c, d) Corresponding spin polarization data and fits of the $\mathrm{x}, \mathrm{y}$ and $\mathrm{z}$ components. Insets show the in-plane polarization vectors obtained from the two-step fit. (e, f) Parts of constant energy surfaces illustrating the measurement positions of the EDCs in reciprocal space. (g, $h$ ) Spin-resolved intensity profiles projected on the quantization axis for the two measured momenta.
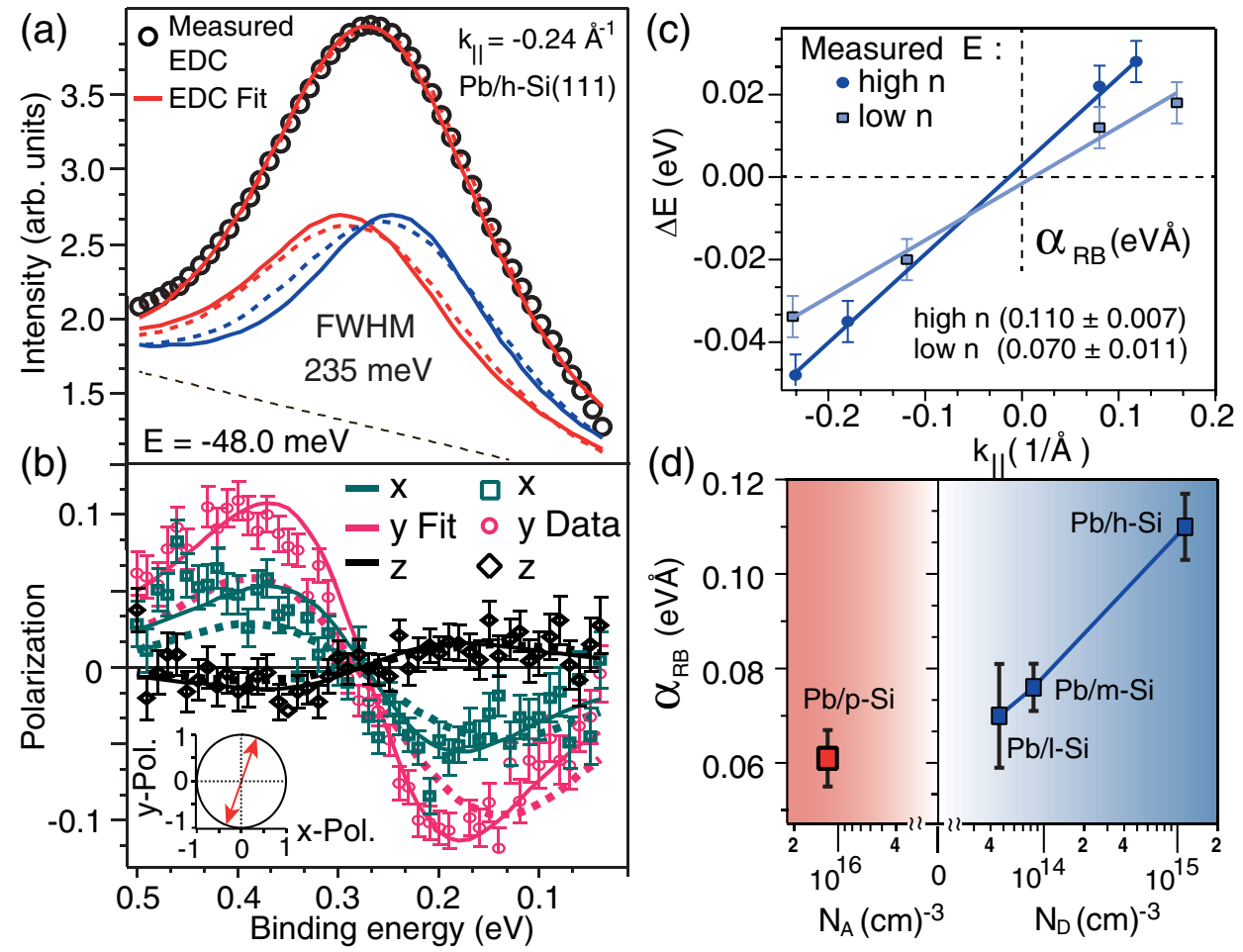

Figure 3 Measured spin-integrated EDC for $\mathrm{Pb} / \mathrm{h}-\mathrm{Si}(111)$ at $\mathrm{k}_{\|}=-0.24 \AA^{-1}$ (a) with Voigt intensity profiles obtained from the two-step fit. (b) Corresponding polarization data and fits of the $\mathrm{x}, \mathrm{y}$ and $\mathrm{z}$ components. The dashed lines are fits performed with $\Delta \mathrm{E}=-33.8 \mathrm{meV}$. (c) Measured $\mathrm{k}$ dependent energy splittings and linear fits (lines) to obtain $\alpha_{R B}$ for $\mathrm{Pb} / \mathrm{h}-\mathrm{Si}(111)$ and $\mathrm{Pb} / \mathrm{l}-\mathrm{Si}(111)$, respectively. (d) Measured $\alpha_{R B}$ vs. doping concentration (left panel) of the heavily p-doped $\mathrm{Si}(111)$ and (right panel) of the differently n-doped $\operatorname{Si}(111)$ substrates. 

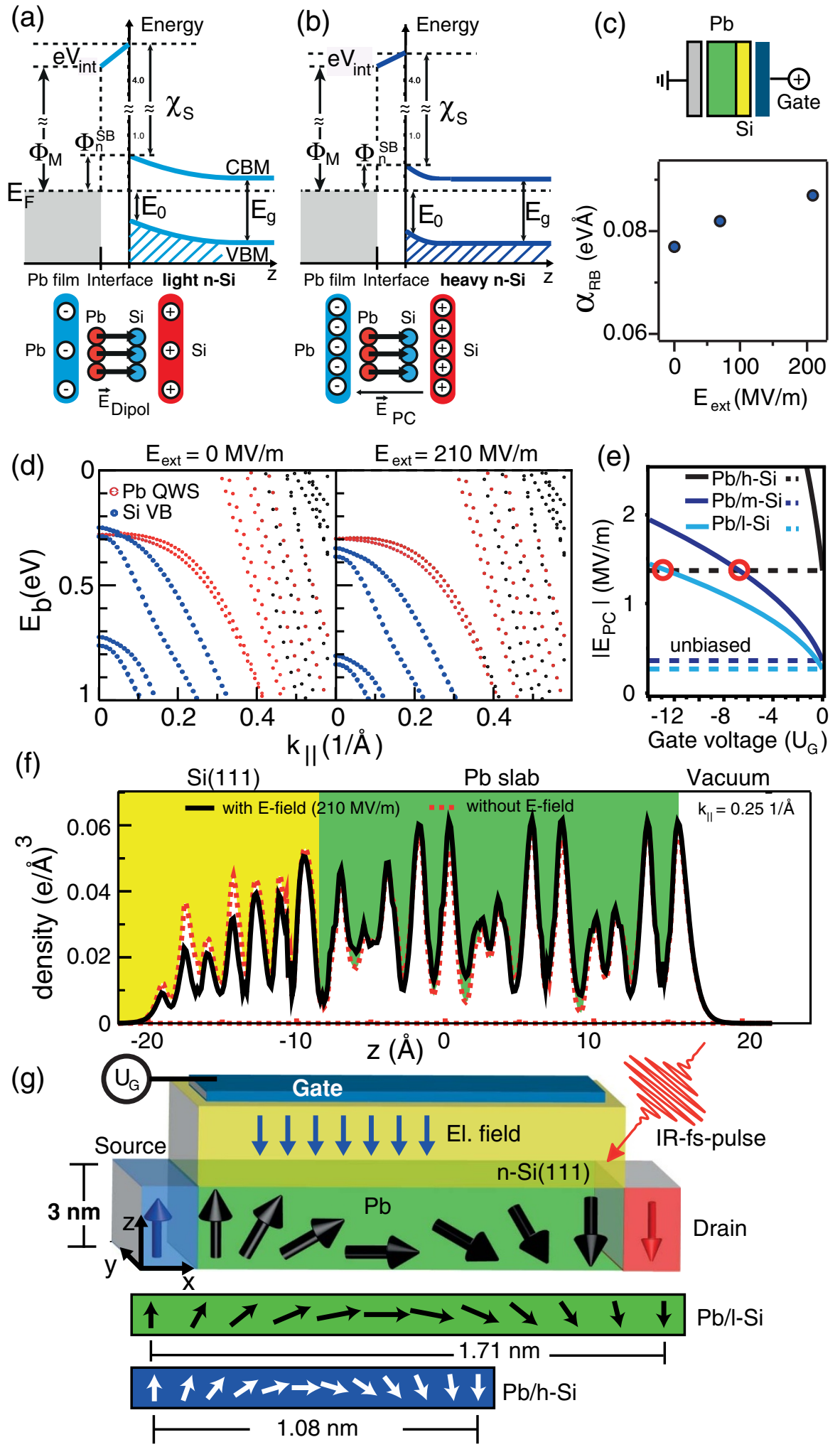

Figure $4 \mid$ (a,b) Energy diagrams of $\mathrm{Pb} / \mathrm{Si}$ within the interface dipole model (not to scale) as a function of $N_{D}$ (upper panels), and capacitor-dipole model (lower panels). (c) $\mathrm{Pb} / \mathrm{Si}$ in a capacitor to illustrate how $E_{\text {ext }}$ is applied to the system (upper panel). Calculated Rashba constant of $\mathrm{Pb} / \mathrm{Si}$ as a function of applied external E-field (lower panel). (d) Influence of $E_{\text {ext }}$ on the energetic position of the Si valence band edge. (e) Electric field in the parallel plate capacitor as a function of (reversed) gate voltage. (f) Charge density plots of QWS in $\mathrm{Pb} / \mathrm{Si}$ with (solid line) and without (dashed line) $E_{\text {ext }}$ at $k_{\|}=0.25 \AA^{-1}$. (g) Sketch of a spin-FET device operated with a gate voltage ( $\left.U_{G}\right)$ or by excitation with femto-second IR pulses (upper panel). Spin precession of $\pi$ for electrons propagating along the $\mathrm{x}$-direction in $\mathrm{Pb}$ films on $\mathrm{l}-\mathrm{Si}(111)$ and h-Si(111) (lower panels). 
energetic distance between the QWS and $E_{0}$ is sensitive to the Schottky barrier $\left(\Phi_{S B}^{n}\right)$ and hence to the donor concentration because both quantities are related via: $\Phi_{n}^{S B}+E_{0}=E_{g}$ where $E_{g}$ is the energy gap of $\mathrm{Si}(111)$.

Our explanation is based on the interface dipole model for Schottky barrier formation, which takes the interaction of the metal and the substrate into account ${ }^{27}$. In this model the formation of polar $\mathrm{Pb}-\mathrm{Si}$ bonds at the interface results in a charge transfer and the establishment of an interface dipole that directly contributes to the $\mathrm{SB}^{27}$ :

$$
\Phi_{n}^{S B}=\Phi_{M}-\chi_{S}+e V_{\text {int }} .
$$

Here $\Phi_{M}$ is the metal work function, $\chi_{S}$ is the electron affinity of the semiconductor, and $V_{\text {int }}$ is the dipole induced voltage drop at the interface. We will argue that the size of $V_{\text {int }}$ is not only determined by the interface chemistry, but also by the doping concentration and the type of dopant (donor or acceptor).

The upper panel of Fig. 4(a) shows the energy band diagram of $\mathrm{Pb} /$ Si deduced from measuring the peak widths of QWSs and QWRs as suggested in Ref. 13, and from core-level shifts of the Si $2 p$ spectrum in the interface region that revealed an accumulation of negative charge at $\mathrm{Si}$ atoms when the $\mathrm{Pb}-\mathrm{Si}$ interface is formed. This is in-line with the predictions of the polarity of the $\mathrm{Pb}-\mathrm{Si}$ bond using the Miedema electronegativity scale and with the study of Si $2 p$ corelevel shifts of $\mathrm{Pb}$ on $\mathrm{n}$ - and $\mathrm{p}$-type $\mathrm{Si}$ that both revealed a shift toward lower binding energies ${ }^{28}$. According to Koopman's initial state model this means that the electric field generated by the interface dipole $\left(\mathrm{E}_{\mathrm{Pb}-\mathrm{Si}}\right)$ points from $\mathrm{Pb}$ to $\mathrm{Si}$ independent of the type of dopant. The lower panel of Fig. 4(a) illustrates our model which explains the influence of the donor concentration on the band edge of $\mathrm{Si}(111)$. It consists of the aforementioned interface dipole placed in a parallel plate capacitor (PC) that generates an electric field of strength ${ }^{29}$ $\left|\mathbf{E}_{\mathrm{PC}}\right|=\sqrt{2 e N_{D}\left(U_{0}+U_{G}\right) / \epsilon \epsilon_{0}}$ at the interface due to the space charge in the depletion layer and hence depends on the density of ionized donors $\left(N_{D}\right) . U_{0}$ is the built-in potential across the Schottky barrier, $U_{G}$ is the gate voltage (for the moment $U_{G}=0 \mathrm{~V}$ ) and $\epsilon=11.9$ the dielectric constant of $\mathrm{Si}$. For $\mathrm{Pb}$ in contact with n-type (p-type) Si the polarity of the capacitor is such that the positive (negative) space charge is on the semiconductor side, while the negative (positive) charge of equal size is balanced on the $\mathrm{Pb}$ side. Hence the direction of the electric field of the dipole and of the capacitor are anti-parallel (parallel) to each other for n-type (p-type) Si. Now, increasing the donor concentration increases the electric field in the capacitor and lowers $V_{\text {int }}$ due to the screening of the interface dipole. Consequently, the SB decreases and the band edge of Si shifts to higher binding energies.

Our finding of a reduction of the SB with increasing $N_{D}$ is consistent with previous results ${ }^{30,31}$. As already pointed out the increased energetic distance between the QWS and $E_{0}$ influences the confinement of the QWS via the metal-substrate phase shift such that the QWS is more strongly localized in the $\mathrm{Pb}$ film, i.e. less charge spills into the substrate. This affects the local charge densities of the QWS wave function with respect to the $\mathrm{Pb}$ cores which determine the spin splitting ${ }^{19}$. We now show how the charge density distribution in the $\mathrm{Pb}$ film changes as a function of the energetic position of the valence band edge using DFT calculations. These calculations were performed on a $10 \mathrm{ML} \mathrm{Pb}$ film on $\mathrm{Si}(111)$ with the in-plane lattice constant of the $\mathrm{Pb}$ film commensurate with that of $\mathrm{Si}(111)$ and with $\mathrm{Pb} /$ Si placed into a parallel plate capacitor producing external electric fields $\left(E_{\text {ext }}\right)$ of various strengths with the positive bias at the Si side, see upper panel of Fig. 4(c). The DFT calculations reveal that increasing $E_{\text {ext }}$ from 0 to $210 \mathrm{MVm}^{-1}$ shifts the Si valence band edge by $\approx 90 \mathrm{meV}$ to higher binding energies, see Fig. 4(d), and simultaneously the calculated Rashba constant increases by $\approx 12 \%$, see lower panel of Fig. $4(\mathrm{c})$. Notice that here $E_{0}$ shifts in the same direction as in the interface dipole model when the donor concentration is increased. As already speculated the increased Rashba constant is a result of an increased localization of the state in the $\mathrm{Pb}$ film, which is apparent from Fig. 4(f) where we show charge density distributions producing a $\alpha_{R B}=0.078 \mathrm{eV} \AA$ at $E_{\text {ext }}=0 \mathrm{MVm}^{-1}$ and $0.087 \mathrm{eVA}$ at $210 \mathrm{MVm}^{-1}$, respectively. This interpretation is also consistent with our results obtained from $\mathrm{Pb} \mathrm{QWS}$ on heavily p-doped $\mathrm{Si}(111)$ where we find the smallest Rashba constant among the studied systems, see left panel of Fig. 3(d) and SOM.

Having shown the sensitivity of the Rashba constant in $\mathrm{Pb}$ QWS to the donor concentration we now discuss the possibility to use $\mathrm{Pb} / \mathrm{Si}$ as a candidate for future spintronic applications. Assuming ballistic spin transport through the Rashba channel we deduce the length (L) at which the injected spin - a coherent superposition of the two orthogonal Rashba spinors - precess by an angle of $\pi$ while propagating along $x$, using $\mathrm{L}=\pi /\left(2 k_{0}\right)$ where $2 k_{0}=2 m^{*} \alpha_{R B} / \hbar^{2}$ is the characteristic Rashba-type momentum splitting deduced from the experiment. For electrons in $\mathrm{Pb} / \mathrm{l}-\mathrm{Si}(111)$ we obtain $\mathrm{L}_{l}=1.71 \mathrm{~nm}$ from $\mathrm{k}_{0, l}=0.092 \AA^{-1}$, and in $\mathrm{Pb} / \mathrm{h}-\mathrm{Si}(111) \mathrm{L}_{h}=1.08 \mathrm{~nm}$ from $\mathrm{k}_{0, h}=$ $0.146 \AA^{-1}$, see lower panels of Fig. $4(\mathrm{~g})$. Such a difference in spin precession length can for example be used to design a spin-field effect transistor (spin-FET). For a spin-FET made of $\mathrm{Pb} / \mathrm{l}-\mathrm{Si}(111)$ with a fixed channel length of only $1.08 \mathrm{~nm}$ this means that changing the voltage drop at the interface by increasing the dipole screening via $\left|\mathbf{E}_{\mathrm{PC}}\right| \propto \sqrt{U_{0}+U_{G}}$ it is possible to go from a low $(\Delta \varphi \approx \pi / 2)$ to a high conducting state $(\Delta \varphi=\pi)$ by ramping $U_{G}$ from 0 to $-12.8 \mathrm{~V}$ as simulated in Fig. 4(e).

In a more general sense, the combination of the possibility to tune the Rashba effect via a gate voltage and the relatively large splitting make Pb QWS on Si an ideal system to study spin-transport effects, such as a varying spin accumulation due to the spin Hall $\mathrm{effect}^{32}$. In this respect it is important to note that an alternative way to vary the spin-splitting may be achieved by optical means - thereby combining spin-based with electro-optical technologies. Recently it has been demonstrated that it is possible to change the SB and correspondingly $E_{0}$ in $\mathrm{Pb} / \mathrm{Si}$ using ultra-short light pulses ${ }^{33}$. This would allow for a study of the time needed to build up or change the spin accumulation and indirectly the spin coherence time. Returning to our device example above, consider a spin-FET in the non-conducting state which can be optically driven. Illuminating the device with fs-pulses leads to a shift of $E_{0}$ such that $k_{0}$ changes and the conducting state is reached. For high-speed applications, the FET should respond quickly to variations of the trigger pulse. In $\mathrm{Pb} / \mathrm{Si}$ a complete builtup of the $E_{0}$ shift is reached within $100 \mathrm{fs}$ and equilibrium is recovered after $600 \mathrm{fs}^{33}$ - the non-conducting state. This yields a switching frequency in the terahertz $(\mathrm{THz})$ regime.

Our results demonstrate how the Rashba-type spin splitting of QWS in ultra-thin $\mathrm{Pb}$ films can be tuned effectively via the donor concentration of the $\mathrm{Si}(111)$ substrate. We conjecture that the energetic position of the Si valence band edge is the decisive factor for this effect. Our results provide the first direct evidence of a doping dependent Schottky barrier. A similar shift of the VB maximum can also be achieved by other means, opening up the possibility to fine-tune the Rashba-type momentum splitting in metallic QWS via a gate voltage or by excitation with ultra-short laser pulses.

\section{Methods}

The ultra-thin $\mathrm{Pb}$ films were prepared in three steps consisting of (i) cleaning of the substrate by flashing above $\mathrm{T}>1300 \mathrm{~K}$ to obtain the $\mathrm{Si}(111)-(7 \times 7)$ reconstruction, (ii) deposition of $\approx 3 \mathrm{ML}$ of $\mathrm{Pb}$ from a Knudsen cell and subsequent annealing at $600 \mathrm{~K}$ for $60 \mathrm{sec}$. to form the dense $(\sqrt{3} \times \sqrt{3}) \mathrm{R} 30^{\circ}(\alpha)$ surface reconstruction $^{34}$, and (iii) deposition of thin crystalline $\mathrm{Pb}$ films at $\mathrm{T}=80 \mathrm{~K}$. The films on both substrates were prepared by depositing the same amount of $\mathrm{Pb}$ with a rate of $\approx 0.3 \mathrm{ML} / \mathrm{min}$. The room temperature substrate resistance $R$ and donor concentrations $\mathrm{N}_{D}$ are for $\mathrm{Pb} / \mathrm{h}$ $\mathrm{Si}(111): \mathrm{R}=4 \Omega \mathrm{cm}, \mathrm{N}_{D}=1.14 \cdot 10^{15} \mathrm{~cm}^{-3}$, for $\mathrm{Pb} / \mathrm{m}-\mathrm{Si}(111): \mathrm{R}=53 \Omega \mathrm{cm}, \mathrm{N}_{D}=$ $8.38 \cdot 10^{13} \mathrm{~cm}^{-3}$ and for $\mathrm{Pb} / \mathrm{l}-\mathrm{Si}(111): \mathrm{R}=95 \Omega \mathrm{cm}, \mathrm{N}_{D}=4.65 \cdot 10^{13} \mathrm{~cm}^{-3}$.

All measurements were performed with the COPHEE end-station at the Swiss Light Source of the Paul-Scherrer-Institute ${ }^{24}$ at a base pressure below $3 \times 10^{-10}$ mbar. The sample temperature during the measurements was $80 \mathrm{~K}$. The energy and angular 
resolution of our spin-resolved (spin-integrated) ARPES measurements were set to $100(25) \mathrm{meV}$ and $\pm 0.75^{\circ}\left(0.5^{\circ}\right)$ in respect of the low efficiency of Mott-scattering. In all measurements a photon energy of $24 \mathrm{eV}$ was used because of the optimal photoemission cross-section for $\mathrm{Pb} \mathrm{QWS}^{35}$.

The ARPES spectrometer is equipped with two orthogonally mounted Mott detectors operated at an acceleration voltage of $40 \mathrm{kV}^{36}$ (see Fig. 1(b)). Each Mott detector measures asymmetries of the photoelectron current backscattered elastically off a polycrystalline gold foil, defined as $A_{i}=\left(I_{R}-I_{L}\right) /\left(I_{R}+I_{L}\right)$, with $I$ being the intensity measured in either right $(\mathrm{R})$ or left $(\mathrm{L})$ detector. The orthogonal arrangement of the two Mott detectors allows to measure asymmetries in $\mathrm{A}_{\tilde{x}}, \mathrm{~A}_{\tilde{y}}$ and $\mathrm{A}_{\tilde{z}}$ directions of the Mott coordinate system $(\tilde{x}, \tilde{y}, \tilde{z})$. Three-dimensional spin polarization data is then obtained by dividing asymmetries by the Sherman function $(S=0.068)$, and by applying a rotation matrix $(\mathrm{T})$ in order to transform the spin polarization components from the Mott reference frame into the sample coordinate system: $P_{i}\left(E_{b}\right)=T\left(S^{-1} A_{\tilde{i}}\left(E_{b}\right)\right)$ with $\mathrm{i} \in(\mathrm{x}, \mathrm{y}, \mathrm{z})$.

We employed density functional theory in the local density approximation ${ }^{37}$, using the full-potential linearized augmented plane-wave method in thin film geometry ${ }^{38}$ (for a program description see www.flapw.de). This allows us to expose the structure, consisting of a ten layer $\mathrm{Pb}$ film placed commensurately on six layers of $\mathrm{Si}(111)$ substrate with a H-terminated backside, to an external electric field. This field is generated by effective condenser plates put in the vacuum at a distance of $5.3 \mathrm{~A}$ above and below the film structure. To simulate the Si-doping, we charge only the condenser plate below the $\mathrm{Si}$ and add the screening charge to the Si substrate.

1. Bychkov, Y. A. \& Rashba, E. I. Properties of a 2D electron gas with lifted spectral degeneracy. JETP Lett. 39, 78 (1984).

2. Dil, J. H. Spin- and angle-resolved photoemission on non-magnetic lowdimensional systems. J. Phys.: Condens. Matter 21, 403001 (2009).

3. Datta, S. \& Das, B. Electronic analog of the electro-optic modulator. Appl. Phys. Lett. 56, 665-667 (1990).

4. Wolf, S. A. et al. Spintronics: a spin-based electronics vision for the future. Science 294, 5546 (2001).

5. King, P. D. C. et al. Large tunable rashba spin splitting of a two-dimensional electron gas in $\mathrm{Bi}_{2} \mathrm{Se}_{3}$. Phys. Rev. Lett. 107, 096802 (2011).

6. Zhu, Z.-H. et al. Rashba spin-splitting control at the surface of the topological insulator $\mathrm{Bi}_{2} \mathrm{Se}_{3}$. Phys. Rev. Lett. 107, 186405 (2011).

7. Eremeev, S. V., Vergniory, M. G., Menshchikova, T. V. \& Chulkov, E. V. The effect of van der Waal's gap expansions on the surface electronic structure of layered topological insulators. New J. Phys. 14, 113030 (2012).

8. Bihlmayer, G., Koroteev, Y. M., Echenique, P. M., Chulkov, E. V. \& Bügel, S. The rashba-effect at metallic surfaces. Surf. Sci. 600, 3888-3891 (2006).

9. Nitta, J., Akazaki, T., Takayanagi, H. \& Enoki, T. Gate control of spin-orbit interaction in an inverted $\operatorname{In}_{0.53} \mathrm{Ga}_{0.47} \mathrm{As} / \mathrm{In}_{0.52} \mathrm{Al}_{0.48} \mathrm{As}$ heterostructure. Phys. Rev. Lett. 78, 1335 (1997)

10. Studer, M., Salis, G., Ensslin, K., Driscoll, D. C. \& Gossard, A. C. Gate-controlled spin-orbit Interaction in a parabolic GaAs/AlGaAs quantum well. Phys. Rev. Lett. 103, 027201 (2009).

11. Koo, H. C. et al. Control of Spin Precession in a Spin-Injected Field Effect Transistor. Science 325, 1515 (2009).

12. Upton, M. H., Wei, C. M., Chou, M. Y., Miller, T. \& Chiang, T. C. Thermal stability and electronic structure of atomically uniform $\mathrm{Pb}$ films on $\mathrm{Si}(111)$. Phys. Rev. Lett. 93, 026802 (2004).

13. Ricci, D. A., Miller, T. \& Chiang, T. C. Chemical tuning of metal-semiconductor interfaces. Phys. Rev. Lett. 93, 136801 (2004).

14. Dil, J. H. et al. Rashba-type spin-orbit splitting of quantum well states in ultrathin Pb films. Phys. Rev. Lett. 101, 266802 (2008).

15. Hoesch, M. et al. Spin structure of the shockley surface state on $\mathrm{Au}(111)$. Phys. Rev. B 69, 241401 (2004)

16. Varykhalov, A. et al. Quantum cavity for spin due to spin-orbit interaction at a metal boundary. Phys. Rev. Lett. 101, 256601 (2008).

17. Rybkin, A. G. et al. Large spin-orbit splitting in light quantum films: Al/W(110). Phys. Rev. B 82, 233403 (2010).

18. He, K. et al. Spin polarization of quantum well states in Ag films induced by the rashba effect at the surface. Phys. Rev. Lett. 101, 107604 (2008).

19. Slomski, B. et al. Manipulating the rashba-type spin splitting and spin texture of Pb quantum well states. Phys. Rev. B 82, 193406 (2011).

20. Mans, A., Dil, J. H., Ettema, A. R. H. F. \& Weitering, H. H. Quantum electronic stability and spectroscopy of ultrathin $\mathrm{Pb}$ films on $\mathrm{Si}(111) 7 \times 7$. Phys. Rev. B 66, 195410 (2002)
21. Upton, M. H., Miller, T. \& Chiang, T.-C. Unusual band dispersion in $\mathrm{Pb}$ films on Si(111). Phys. Rev. B 71, 033403 (2005).

22. Dil, J. H., Kim, J. W., Kampen, Th., Horn, K. \& Ettema, A. R. H. F. Electron localization in metallic quantum wells: $\mathrm{Pb}$ versus In on Si(111). Phys. Rev. B 73, 161308 (2006).

23. Slomski, B., Meier, F., Osterwalder, J. \& Dil, J. H. Controlling the effective mass of quantum well states in $\mathrm{Pb} / \mathrm{Si}(111)$ by interface engineering. Phys. Rev. B 83, 035409 (2011).

24. Hoesch, M. et al. Spin-polarized fermi surface mapping. J. Electron. Spectrosc. Relat. Phenom. 124, 263-279 (2002).

25. Meier, F., Dil, J. H. \& Osterwalder, J. Measuring spin polarization vectors in angleresolved photoemission spectroscopy. New J. Phys. 11, 125008 (2009).

26. Chiang, T.-C. Photoemission studies of quantum well states in thin films. Surf. Sci. Rep. 39, 181-235 (2000).

27. Tung, R. T. Chemical bonding and fermi level pinning at metal-semiconductor interfaces. Phys. Rev. Lett. 84, 6078-6081 (2008).

28. Le Lay, G. \& Hricovini, K. Comment on Atomic-structure-dependent schottky barrier at epitaxial Pb/Si(111) interfaces. Phys. Rev. Lett. 65, 807-807 (1990).

29. Brillson, L. Contacts to Semiconductors - Fundamentals and Technology. Noyes Publications (1993)

30. Kang, M.-S. et al. Metal work-function and doping-Concentration dependent barrier height of Ni-contacts to $4 \mathrm{H}-\mathrm{SiC}$ with metal-embedded nano-particles. Material Science Forum 717-720, 857 (2012).

31. Xieqiu, Z. et al. Effect of substrate doping concentration on quantum well states of $\mathrm{Pb}$ island grown on Si(111). Surf. Sci. 604, 175-180 (2010).

32. Hirsch, J. E. Spin Hall Effect. Phys. Rev. Lett. 83, 1834-1837 (1999).

33. Rettig, L., Kirchmann, P. S. \& Bovensiepen, U. Ultrafast dynamics of occupied quantum well states in $\mathrm{Pb} / \mathrm{Si}(111)$. New Journal of Physics 65, 023047 (2012).

34. Weitering, H. H., Heslinga, D. R. \& Hibma, T. Structure and growth of epitaxial $\mathrm{Pb}$ on Si(111). Phys. Rev. Lett. 45, 5991-6002 (1992).

35. Dil, J. H., Kim, J. W., Gokhale, S., Tallarida, M. \& Horn, K. Self-organization of Pb thin films on $\mathrm{Cu}(111)$ induced by quantum size effects. Phys. Rev. B 70, 045405 (2004).

36. Petrov, V. N., Grebenshikov, V. V., Grachev, B. D. \& Kamochkin, A. S. New compact classical $40 \mathrm{kV}$ Mott polarimeter. Rev. Sci. Instrum. 74, 1278-1281 (2003).

37. Perdew, J. P. \& Zunger, A. Self-interaction correction to density-functional approximations for many-electron systems. Phys. Rev. B 23, 5048 (1981).

38. Krakauer, H., Posternak, M. \& Freeman, A. J. Linearized augmented plane-wave method for the electronic band structure of thin films. Phys. Rev. B 19, 1706 (1979).

\section{Acknowledgements}

We thank C. Hess, F. Dubi, M. Kropf and S. Stutz for technical support and G. Salis for discussion of the results. This work was supported by the Swiss National Foundation.

\section{Author contributions}

J.H.D. designed the research. B.S., G.L. and J.H.D. performed the experiments and analysis of data. B.S., J.H.D., G.L., J.O. wrote the manuscript. G.B. performed the density functional theory calculations. All authors reviewed the manuscript

\section{Additional information}

Supplementary information accompanies this paper at http://www.nature.com/ scientificreports

Competing financial interests: The authors declare no competing financial interests.

How to cite this article: Slomski, B., Landolt, G., Bihlmayer, G., Osterwalder, J. \& Dil, J.H. Tuning of the Rashba effect in $\mathrm{Pb}$ quantum well states via a variable Schottky barrier. Sci. Rep. 3, 1963; DOI:10.1038/srep01963 (2013).

This work is licensed under a Creative Commons AttributionNonCommercial-NoDerivs Works 3.0 Unported license. To view a copy of this license, visit http://creativecommons.org/licenses/by-nc-nd/3.0 